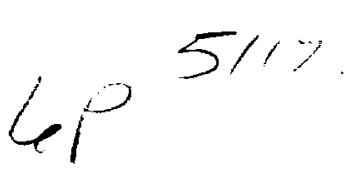

\title{
A Hardware-in-the-Loop Testbed for Spacecraft Formation Flying Applications*
}

\author{
Jesse Leitner \\ NASA Goddard Space Flight Center \\ Code 571 \\ Greenbelt, MD 20771 \\ (301) 286-2630 \\ jesse.leitner@gsfc.nasa.gov
}

Abstract- The Formation Flying Test Bed (FFTB) at NASA Goddard Space Flight Center (GSFC) is being developed as a modular, hybrid dynamic simulation facility employed for end-to-end guidance, navigation, and control (GN\&C) analysis and design for formation flying clusters and constellations of satellites. The FFTB will support critical hardware and software technology development to enable current and future missions for NASA, other government agencies, and external customers for a wide range of missions, particularly those involving distributed spacecraft operations. The initial capabilities of the FFTB are based upon an integration of high fidelity hardware and software simulation, emulation, and test platforms developed at GSFC in recent years; including a high-fidelity GPS simulator which has been a fundamental component of the Guidance, Navigation, and Control Center's GPS Test Facility. The FFTB will be continuously evolving over the next several years from a tool with initial capabilities in GPS navigation hardware/software-in-the-loop analysis and closed loop GPS-based orbit control algorithm assessment to one with cross-link communications and relative navigation analysis and simulation capability. Eventually the FFTB will provide full capability to support all aspects of multi-sensor, absolute and relative position determination and control, in all (attitude and orbit) degrees of freedom, as well as information management for satellite clusters and constellations. In this paper we focus on the architecture for the FFTB as a general GN\&C analysis environment for the spacecraft formation flying community inside and outside of NASA GSFC and we briefly reference some current and future activities which will drive the requirements and development.

\section{TABLE OF CONTENTS}

1. INTRODUCTION

2. FFTB SYSTEM ARCHITECTLRE

3. TECHNOLOGY PROGRAM SLPPORT

4. MISSION SLPPORT

5. FUtURE Directions and CONClusions

\section{INTRODUCTION}

Formation flying and distributed spacecraft missions are becoming the wave of the future for NASA and the United States Department of Defense (DoD). Dozens of missions have been formulated which exploit distributed spacecraft technologies to enable higher resolution imagery and interferometry, robust and redundant fault-tolerant architectures, and complex networks dispersed over clusters of satellites in space. Figure 1 shows a current list of missions that are proposed or funded in which NASA has the management responsibility or significant involvement.

In order to support these missions starting with initial concept definition, NASA Goddard Space Flight Center's (GSFC's) Guidance, Navigation. and Control (GN\&C) Center has been developing a Formation Flying Testbed (FFTB) to bridge the gap between component-level formation flying technology development projects and the upcoming distributed spacecraft programs. The FFTB is being developed as a modular, hybrid dynamic simulation facility for end-to-end guidance, navigation, and control (GN\&C) analysis and design for formation flying clusters and constellations of satellites. The FFTB will support critical hardware and software technology development to enable current and future missions for NASA, other government agencies, and external customers for a wide range of missions, particularly those involving distributed spacecraft operations. The initial capabilities of the FFTB are based upon an integration of high fidelity hardware and software simulation. emulation, and test platforms developed or employed at GSFC in recent years, including a highfidelity Global Positioning System (GPS) simulator which has been a fundamental component of the GN\&C Center's GPS Test Facility. The FFTB will be continuously evolving over the next several years from a tool with initial capabilities in GPS navigation hardware/software-in-the-loop analysis and closed loop GPS-based orbit control algorithm assessment to

* L.S. Government work not protected by U.S. copyright. 


\begin{tabular}{|c|c|c|}
\hline $\begin{array}{l}\text { Projected } \\
\text { Launch Year }\end{array}$ & Vission Vame & Hission Type \\
\hline$|k|$ & Now Villennium Program INMPIlio-1 & Earth Science \\
\hline 111 & (iraviry Recovery and Climate Recovery (CRAACE) & Earth Sicience \\
\hline 112 & Univenity Nanosal va ir Foree Reseurch Laboritory Launch I & Technology Demonstrator \\
\hline 112 & Universily Nanosals/Air Force Revearch Lahoritory Luunch 2 & Technology Demonstralor \\
\hline 013 & Aurural Lites Mission (MIDEX) & Space Sciences SEC \\
\hline 913 & Fechsat-21 Demonstrator AFRL & Technology Demo \\
\hline 113 & New Millennium Proyram (NMP) ST-5 & Space Sciente \\
\hline 14 & DARPA/AF Discoverer II & Technology Demo \\
\hline 05 & Origins Proyram Space Technology-3 & Space Science ASO \\
\hline 05 & Magnetospheric Multiscale (MMS) & Space Science SEC \\
\hline 05 & Space Interterometry Mission (SIM) & Space Science/ASO \\
\hline 107 & Global Precipitation Mission (EOS-9) & Earth Science \\
\hline 08 & Gospace Electrodynamic Connections $[\mathrm{GEC}]$ & Space Science SEC \\
\hline 08 & Constellation $-\bar{X}$ & Space Science SEU \\
\hline 08 & Laser Interterometric Space Antenna (LiSA) & Space Science/SEU \\
\hline 08 & Radiation Belt Mappers & Space Science SEC \\
\hline (n) & Ionospheric Mappers & Space Science SEC \\
\hline 09 & DARWIN Space Infrared Interferometer European Space Agency & Space Science \\
\hline 10) & Magnetospheric Constcllation (DRACO) & Space Science/SEC \\
\hline 10 & Leonardo Bi-direction Radiant Forcing (GSFC) & Earth Science \\
\hline (15t & Stellar Imager and Seismic Probe (SISP) & Space Science/SEC \\
\hline $05+$ & Astronomical Low Frequency Array (ALFA)/Explorers & Space Science \\
\hline $0 \mathrm{St}$ & Sentinels & Space Science/SEC \\
\hline $05+$ & Soil Moisture and Ocean Salinity Observing Mission (EX-4) & Earth Science \\
\hline $05+$ & Time-Dependent Gravity Field Mapping Mission (EX-5) & Earth Science \\
\hline $05+$ & Vegetation Recovery Mission (EX-6) & Earth Science \\
\hline $105+$ & Cold Land Processes Rescarch Mission (EX-7) & Earth Science \\
\hline $05+-$ & Submillimeter Probe of the Evolution of Cosmic Structure (SPECS) & Space ScienceiSEU \\
\hline $15+$ & MAXIM X-ray Interferometry Mission & Space Science SEU \\
\hline $15+$ & Terrestrial Planct Mapper & Space Science/ASO \\
\hline 15 r & ITM Waves Outer Heliospheric Radio Imager (OHRI) & Space Science/SEC \\
\hline $15+$ & NASA Goddard Space Flight Center Earth Sciences Vision & Earth Science \\
\hline $15+$ & NASA Institute of Advanced Concepts/Very Large Optics for the Study & Space Science \\
\hline $15+$ & NASA Institute of Advanced Concepts (Ultra-high Throughput X-Ray & Space Science \\
\hline $15+$ & $\begin{array}{l}\text { NASA Institute of Advanced Concepts /Structureless Extremely Large } \\
\text { Yct Very Lightweight Swarm Array Space Telescope }\end{array}$ & Space Science \\
\hline
\end{tabular}

Figure 1: Distributed Spacecraft Mission List

one with cross-link communications and relative navigation analysis and simulation capability. Eventually the FFTB will provide full capability to support all aspects of multi-sensor. absolute and relative position determination and control, in all (attitude and orbit) degrees of freedom, as well as information management for satellite clusters and constellations.

The remainder of this paper is organized as follows. The next section describes the general FFTB system architecture which is designed to ensure a wide applicability to virtually all of the upcoming distributed spacecraft missions, as well as the technology programs that feed in. After that, two key formation flying technology programs are described which are driving the current requirements for the FFTB. This will then be followed by a synopsis of future directions and concluding remarks.

\section{SYSTEM ARCHITECTURE}

In the development of a simulation or emulation testbed. it is important to consider the general utility to of the infrastructure to support the widest range of relevant programs. With this in mind, the first step in planning the FFTB was to define an architecture which ensures maximum general utility. This is portrayed in Figure 2.
This diagram defines the system architecture in its most general form, without respect to whether centralized or decentralized navigation or control are being employed. The components are defined as follows.

1 Central Simulation Controller (CEN) - this is the underlying software and hardware infrastructure that monitors all subsystem activities. This provides the "God"s eye" view of the testbed. The CEN is composed of a spacecraft formation component and a laboratory simulator component. The spacecraft formation component is composed of the elements of the spacecraft command and data handling $(\mathrm{C} \& D H)$ and communication subsystems which are not accounted for in the individual spacecraft on-board processors. The laboratory simulator component is the infrastructure that combines all of the components together in the lab, receives terminal inputs, sends display data. and connects the testbed to outside facilities.

2 Intersatellite Communications Subsystem (ICS) - consists of all hardware and software components related to the satellite crosslinks for both comm and ranging. This system also handles the 


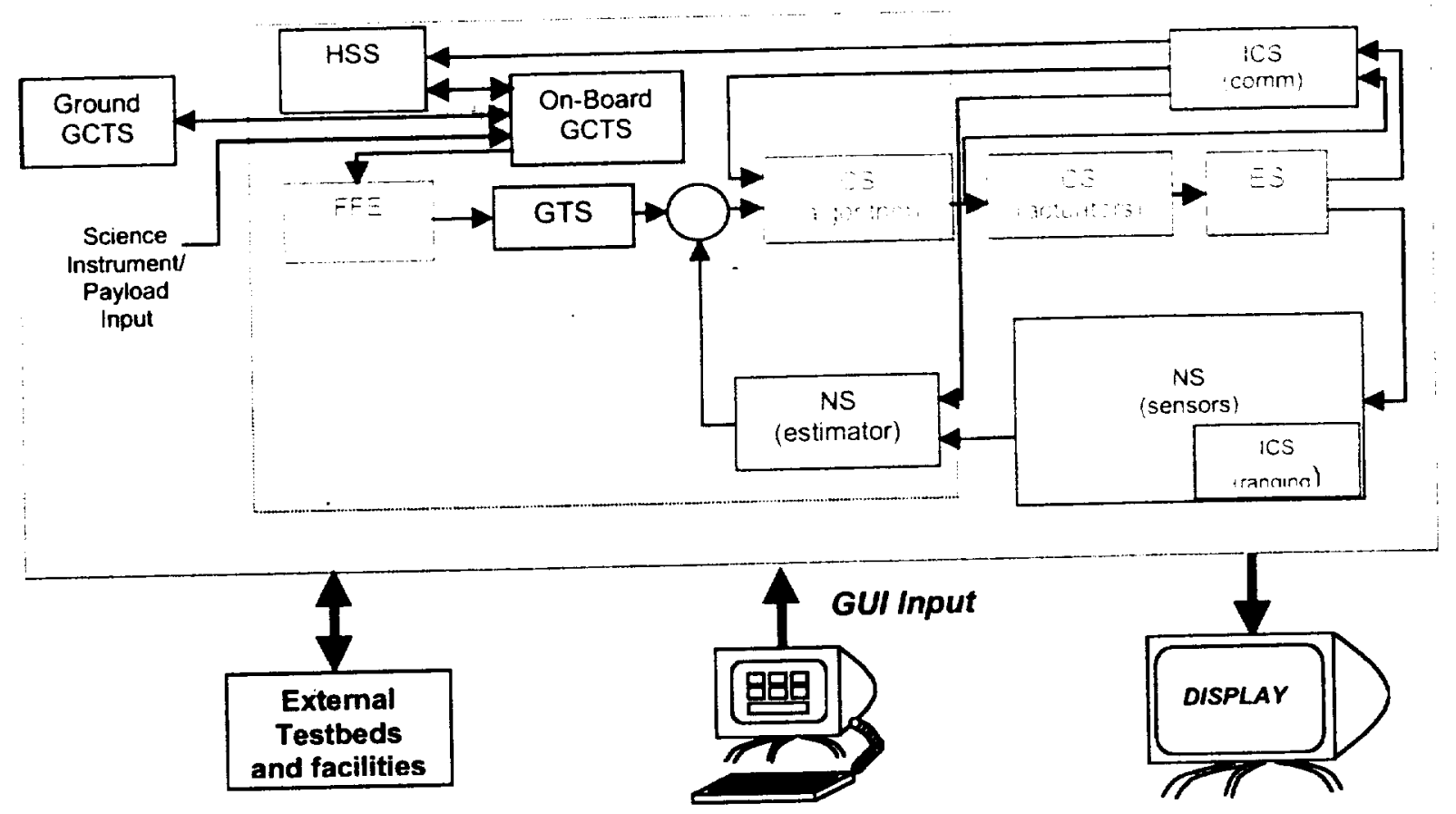

Figure 2: FFTB System Architecture

on-board intersatellite C\&DH of the formation.

3 On-board Processing Subsystem (PS) - consists of all software and hardware representing onboard processing functions on the spacecraft.

4 Ground Control and Telemetry Subsystem (GCTS) - consists of all on-ground control and data transmission functions. The on-board GCTS simply represents an on-board transition of traditional ground-based operations functions.

5 Navigation Subsystem (NS) - consists of all hardware (sensors, GPS receivers and antennas, etc), models and simulations thereof, and software (estimators, etc) involved in providing estimated state vectors. The software component lies entirely on the on-board processing system (PS).

6 Guidance and Trajectory System (GTS) - holds and computes the maneuver plans for the individual satellites in the cluster.

7 Environment Subsystem (ES) - consists of the models of the spacecraft dynamics (orbit, attitude. possibly flexible modes) and space environment. including the ionosphere and atmosphere. Additionally, this includes any sensor noise models as well as the motion of the GPS satelites, where applicable.

8 Vehicle Control Subsystem (CS) - consists of all hardware (actuation devices), models and simula- tions thereof, and software (control laws, etc) involved in computing forces and moments applied to each individual vehicle.

9 Health and Status Subsystem (HSS) - these are the on-board processing items which track system mnemonics, look for out-of-range variables, perform fault-detection, and, in general, perform higher-level control on the vehicle via agents or other mechanisms to enable vehicle and cluster autonomy.

10 Formation Flying Executive (FFE) - this provides the connectivity between the desired motion of the virtual platform and that of the individual satellites. This is an on-board software component that will take a high-level virtual platform guidance command and allocate the guidance to each individual vehicle. In the simplest form, it defines the initial conditions of each satellite based on a desired cluster configuration.

The general architecture defined above is a guideline for the FFTB used to ensure the general capability to integrate a component or set of components into the testbed for integrated system analysis. This ensures the ability to solve required customer problems while simultaneously building infrastructure and capability to support vastly differing formation flying projects and/or programs. While the figure is a simple block diagram incorporating standard GN\&C elements, it is important to point out that this representation is unique in 
defining a new architecture for controlling spacecraft formations in which we view the system as a cluster as opposed to several independent, individual spacecraft. This is an essential view for those missions which require the cluster(s) of spacecraft to act as a single entity. such as a synthetic aperture of a telescope or a multiple spacecraft Michelson interferometer. There are several driving factors for defining such an architecture. First, in most cases the spacecraft must continuously control position and orientation either relative to the other spacecraft, or at the very least in consideration of the actions of the other spacecraft. This is a major transition from typical current spacecraft operations in which the attitude may be maintained or controlled autonomously on-board, but the orbit is monitored from the ground and on a weekly, monthly, or longer scale; corrected through a ground-based effort (coordinated between independent attitude control and orbit maintenance groups) involving significant time and manpower for planning and scheduling the maneuvers. In short, corrections that would have occurred on the scale of weeks or months must now be done, in some cases, in a fraction of a second and without ground intervention. The orbit control relies on both good sensory information and proper control action with respect to the vehicle attitude in order to do its own job. This gives rise to a more coupled view, both at the intra- and inter-spacecraft level, such that attitude and orbit determination (AOD) and attitude and orbit control ( $A O C$ ) are respectively coupled and termed simply "navigation" and "control" respectively, with much more general definitions than traditionally employed. Another important consideration is that while now there is a "cluster-level" control of the virtual platform, the bulk of the sensing and control is actually implemented at the individual spacecraft level. This gives rise to the FFE as defined above as well as a slew of functions providing higher-level control and oversight to the spacecraft, which are encompassed in the HSS and the on-board GCTS. The result is now a series of software elements which are taking the place of large ground support teams previously required for major satellite programs. The FFTB has the role of defining these formation flying architectures, validating them in a realistic environment, and providing the means to transition them to demonstrational and operational use in space.

\section{TECHNOLOGY PROgRAM SUPPORT}

The following subsections briefly describe the two programs funded by the NASA Explorers program and the Space Operations Management Office (SOMO) which are driving the initial requirements for FFTB capability. The first is a Decentralized Formation Control effort [ 1$]$ and the second is an effort in GPS-based relative navigation for high altitude earth orbiting satellites [2]. Using the funding and efforts that these projects would have used to independently perform modeling and simulation analysis, the initial capabilities for the FFTB have been developed such that infrastructure is available for a range of other related projects and, most importantly, the tools are available to transition to the upcoming distributed spacecraft thight programs at Goddard and elsewhere.

\section{Decentralized Control}

One of the first projects defining FFTB requirements and driving schedule and milestones is a decentralized formation control algorithm development activity funded by the NASA Explorers program. The methodology is an innovative application of well-known Linear Quadratic Gaussian (LQG) control theory implemented over a distributed cluster of vehicles. This project will result in a high-fidelity GPS-based implementation of decentralized formation control in the FFTB by June of 2001 . Figure 3 portrays the specific implementation of the integrated closed-loop analysis.

The key elements represented in this figure are (1) an environment simulation which is composed of $2-4$ vehicle orbital dynamic models running in a software tool developed by the Hammers Corporation called VirtualSat Pro (or VSat Pro, for short); (2) a Global Simulation Systems (GSS) 4760 dual frequency, 4 RF output (each with 16 channels), GPS RF signal simulator, which is driven through an IEEE 488 interface from the VSat Pro model; (3) 2-4 in-house developed, openarchitecture "PiVoT" GPS receivers, each running an extended Kalman Filter developed at NASA Goddard entitled GEODE (GPS-enhanced Orbit Determination)[4] producing absolute navigation solutions; (4) 2-4 rack-mounted PCs, communicating with each other through an ethernet hub, each running formation command and data handling $(\mathrm{C} \& D H)$, a relative navigation version of GEODE, as well as the decentralized control law; and (5) a Cesium timing source to ensure proper synchronization of all of the components, (6) a NASA developed tool commercialized by the Hammers Co. called ITOS (Integrated Test and Operations System) used to provide simulation of the ground control interface. This demonstration will validate a formation navigation and control strategy applicable to a wide range of existing and proposed upcoming distributed spacecraft missions within NASA and the DoD and will establish an infrastructure to provide analysis support to a multitude of upcoming missions. Additionally, this will be used as the first increment to the FFTB architecture. In reference to Figure 2, iterns (1). (2), and (5) above constitute the ES in this implementation. Item ( 3 ) is the NS, with the receivers being the sensor component and GEODE being the estimator component. Item (4) represents the PS and communication component of the ICS. In this implementation the CEN is simply represented by a series of PCs which interface with various components of the simulation. Lastly, ITOS maps into the Ground GCTS.

\section{High Altitude Relative Navigation}

The current driving force behind the FFTB is an effort which extends the capabilities of the GEODE software on the PiVoT open architecture receiver to develop GPS-based relative navigation capabilities for satellite formations which are (or have significant regions) well above the GPS constellations. such as in highly-elliptical orbits or geosynchronous orbits. The effort essentially involves a coordination between the tracking loops in the receiver and the on-board extended Kalman filter, 


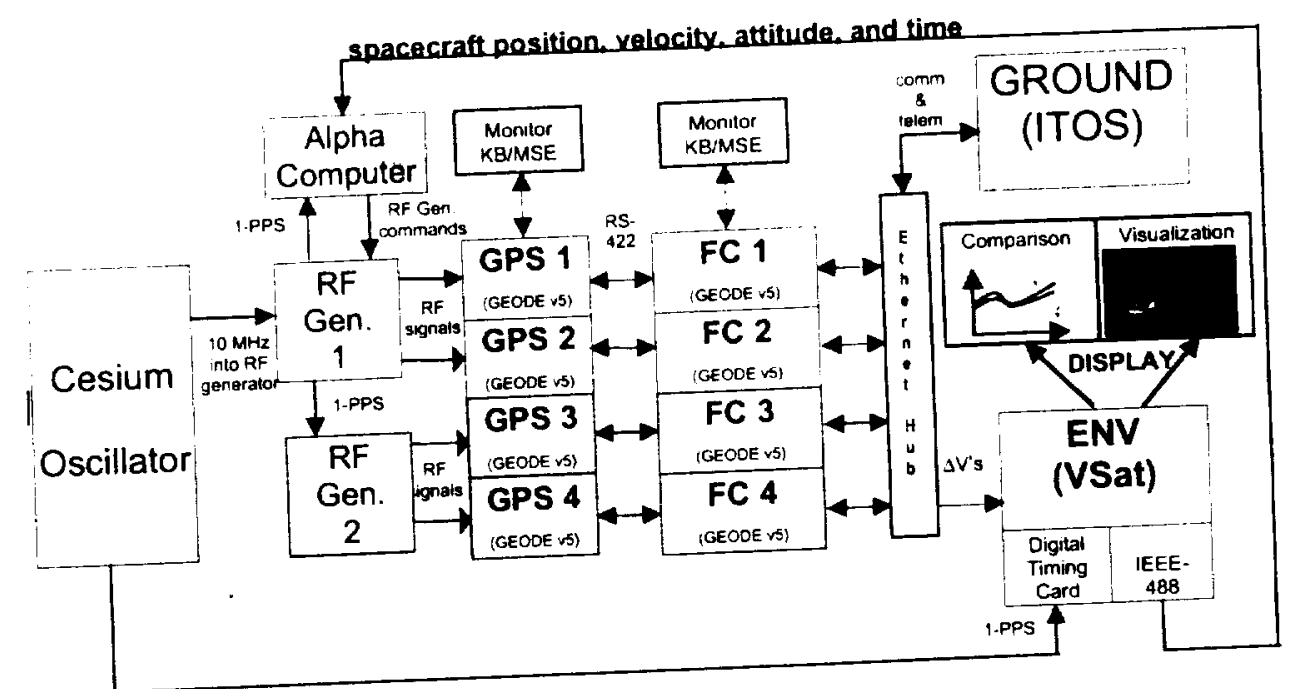

Figure 3: FFTB Decentralized Control Implementation

as well as an extremely high-fidelty modeling of the dynamics in GEODE to enable propagation during long periods without tracking any GPS satellites. This project is fully enabled by the high fidelity integrated GPS simulation capability of the FFTB as well as the open-architecture provided by the PiVoT GPS receivers, as conventional, off-the-shelf receivers do not provide the capability to modify the code in the on-board processor or the tracking loops.

\section{MISSION SUPPORT}

While the support to technology programs has been the driving force behind developing the FFTB, the actual existence is necessitated by upcoming spacecraft missions, such as those listed in Figure 1. In particular, the FFTB has begun to work closely with several of these missions to support preliminary analysis for the programs as well as to ensure that the infrastructure developed by current testbed activities provides a general enough platform to support key distributed spacecraft programs. The implementation of technologies in the FFTB is currently performed with direct application to some of the missions in which GPS is likely to be the primary navigation sensor (University NanoSats, TechSat21, Leonardo) as well as some where GPS is a good candidate but where the performance at the extreme altitudes is still open to question (Magnetospheric Multiscale or MMS). Furthermore, with longer-term capabilities in mind, such programs such as the Laser Interferometer Space Antenna (LISA) [3] and the Stellar Interferometer are driving FFTB infrastructure outside of the realm of GPS-based navigation by being in proposed heliocentric and Earth-Sun Lagrange points, respectively. Henceforth, a major element of the FFTB activity is the interaction with the key investigators of upcoming distributed spacecraft missions and the subsequent insertion of requirements into the FFTB architecture and development plan. It is essential that the requirements and specific GN\&C elements of these missions which are further out be considered early on to ensure that the capa- bility is there for detailed support when the proper time comes.

\section{FUTURE DIRECTIONS AND CONCLUSIONS}

A general system architecture for NASA Goddard's GNCC Formation Flying Testbed was presented, followed by a specific implementation within that architecture geared to validate a current formation control algorithm being developed at GSFC. As the emphasis of the two Explorers efforts are on GPS-based relative navigation and control, several of the elements of the architecture are not required for some of the nearterm upcoming demonstrations. For example, the intersatellite communication system is trivially modeled as a TCP/IP communication network through an ethernet hub and the ranging will be computed through filtered differencing of the GPS measurements. At some time shortly following this demonstration, the upcoming programs will drive the requirements towards a more realistic implementation involving an actual flight-like intersatellite communication and ranging system as well as an associated channel model, geared to the FFTB, analogous to the GPS RF signal simulator/GPS Receiver combination currently employed. Likewise, communication with the ground and NASA's Tracking Data Relay Satellite System (TDRSS) will become important. Henceforth, the FFTB is headed towards a full RF emulationisimulation of the formation flying problem as shown in Figure 4. Finally, the plans are to open up the FFTB to technology and mission level customers through a web-based scheduling and validation process which will be available sometime in late 2001

\section{REFERENCES}

[1] Carpenter. J.R., "A Preliminary Investigation of Decentralized Control for Satellite Formations." Presented at the 2000) IEEE Aerospace Conference. Big Sky. MT. Mar. 2000 . 


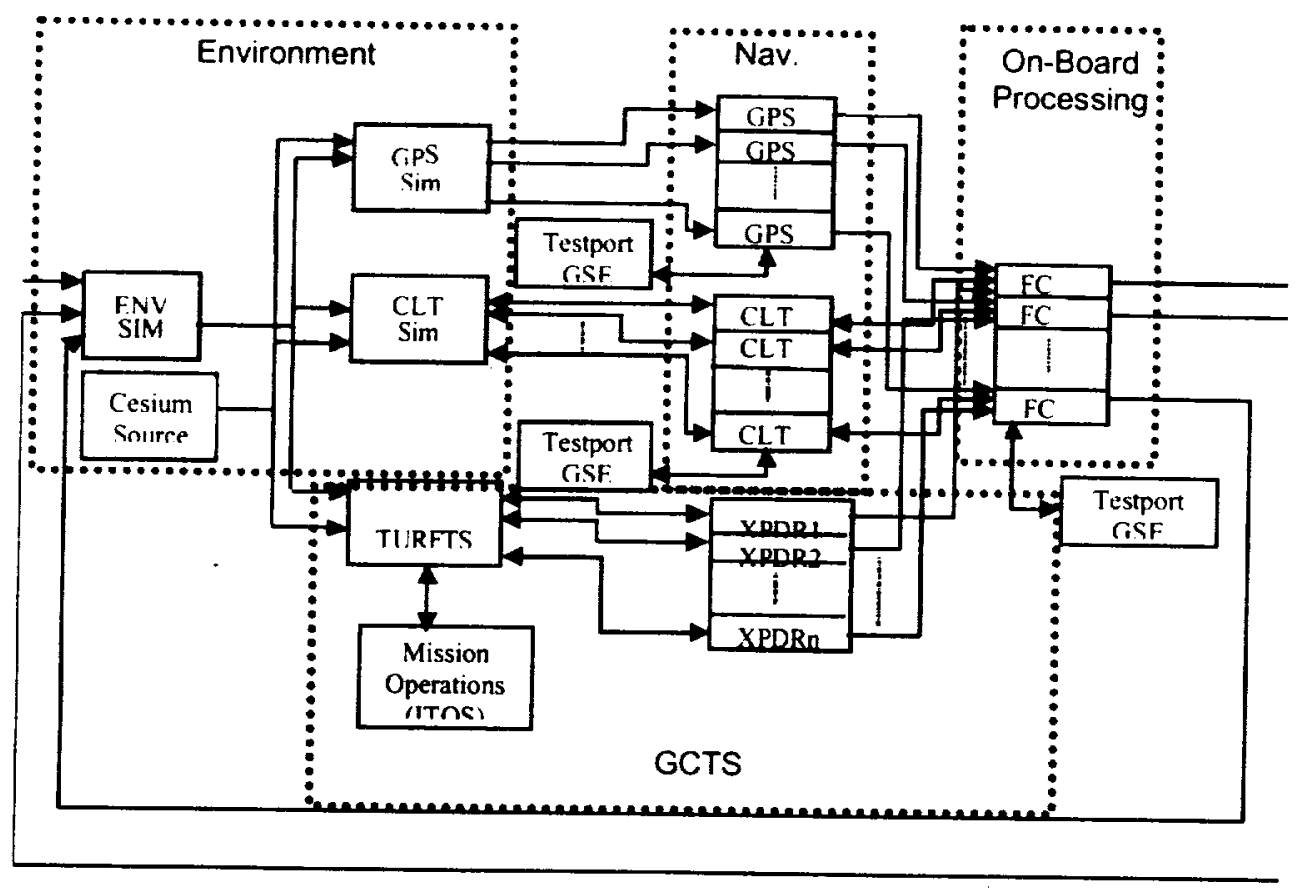

Figure 4: FFTB Full RF Implementation

[2] Garrison, J., Axelrad, P., "Relative Navigation in Elliptical Orbits Using an Iterative Nonlinear Filter," Presented at the Institute of Navigation Conference, Albuquerque, NM, Sept. 1999.

[3] LISA study team, "LISA Pre-Phase A Report," Second Edition, Max-Planck Institut fur Quantenoptik Report MPQ 233, July 1998.

[4] T. Lee and A. Long, "Global Positioning System Enhanced Orbit Determination Mathematical Specifications," CSC-96-932-05R0UDI, Ver. 4, Udate I., July 1999.

Jesse Leitner is a Guidance. Navigation, and Control Systems Engineer at NASA Goddard Space Flight Center, where he has worked in the Guidance, Navigation, and Control Center since January of 2000. He is the technical director of the GN\&C Formation Flying Testbed. His current research areas involve formation flying GN\&C development, integrated GN\&C performance analysis, and nonlinear control system design. Prior to coming to NASA Goddard, Dr. Leitner was at the Air Force Research Laboratory. Space Vehicles Directorate from 1993 1999, where he led research and development efforts in Space Flight Dynamics and Control and analysis of imaging and signal intelligence systems, including advanced modeling and simulation development for a range of satellite remote sensing systems. Dr. Leitner received his B.S. in Aerospace Engineering from The University of Texas at Austin in 1990 and the MS and PhD in Aerospace Engineering from Georgia Tech in 1992 and 1995, respectively. 